\title{
Perfect Absorption in Ultra-thin Uniform and Nanostructured Media
}

\author{
C. Martijn de Sterke ${ }^{1}$, Björn C.P. Sturmberg ${ }^{1}$, Lindsay C. Botten ${ }^{2,3}$, Christopher G. Poulton ${ }^{3}$, Kokou B. \\ Dossou $^{3}$, Ross C. McPhedran ${ }^{1}$
}

1: Centre for Ultrahigh Bandwidth Devices for Optical Systems (CUDOS), School of Physics, University of Sydney, Sydney, Australia 2: National Computational Infrastructure, Australian National University, Canberra, Australia 3: Centre for Ultrahigh Bandwidth Devices for Optical Systems (CUDOS), School of Mathematical and Physical Sciences, University of Technology Sydney, Sydney, Australia martijn.desterke@sydney.edu.au

\begin{abstract}
We show that perfect absorption can be achieved in ultra-thin gratings composed of weakly absorbing dielectric materials combined with a mirror. The structures can be fabricated using standard processing techniques.

OCIS codes: (050.1950) Diffraction gratings, (040.5160) Photodetectors, (040.5350) Photovoltaics
\end{abstract}

\section{Introduction}

Strongly absorptive, ultra-thin films may create new opportunities for high signal-to-noise ratio photodetectors and efficient photovoltaics. Reducing the volume of active material increases the open circuit voltage of a solar cell and reduces the dark current noise of photodetectors. Using ultra-thin films enables the use of materials with poor electronic properties, in which the diffusion length of the absorption generated electron-hole pairs limits the film thickness. Aside from applications, the achievement of perfect absorption is an intriguing problem in its own right.

\section{Uniform films}

It is well known that a uniform layer of thickness $d$ and (complex) refractive index $n=n^{\prime}+i n^{\prime \prime}$, placed between semi-infinite media and which is thin (i.e, $n d \ll \lambda$ ) can absorb at most $50 \%$ of light if it is incident from one side [1]. Perfect absorption can be achieved if the light is incident from both sides, or if the layer has a perfect mirror on one side (and has a thickness $d / 2$ ). The condition for this to be achieved is

$$
r+\delta=0,
$$

where $r=(m-n) /(m+n)$, with $r$ the Fresnel coefficient for light coming from the external medium, which is taken to have the (real) refractive index $m$, and $\delta=e^{i k_{0} n d}$, where $k_{0}$ is the vacuum wavenumber, describes the propagation through the layer. Condition (1) corresponds to that for critical coupling, where the $Q$-factors associated with absorption in the layer and for coupling out of the layer are the same [2,3]. For normal incidence we find that condition (1) leads to $n=\sqrt{2 i m /\left(k_{0} d\right)}$ as $d \rightarrow 0$ [1]. Thus in this limit, in which the film layer is thin, its refractive index lies on the diagonal where $n^{\prime}=n^{\prime \prime}$. This is illustrated in Fig 1a, which shows the absorption of $\lambda=700 \mathrm{~nm}$ light in a layer with $d=10 \mathrm{~nm}$ in air versus $n^{\prime}$ and $n^{\prime \prime}$. Full absorption can be achieved on the diagonal, consistent with the above analysis. However, for this solution $n^{\prime}=n^{\prime \prime}$ which requires quite unusual materials [5].

\section{Structured films}

Different approaches have been proposed to achieve perfect absorption at more convenient refractive indices [4-7]. Many of these involve metamaterials or plasmonic effects and they therefore involve metals. Structures that operate in the microwave, terahertz and infrared regimes have been demonstrated [7], but extending this to shorter wavelengths is a challenge because of fabrication challenges and because parasitic losses due to the presence of the metals. Our approach involves the inclusion of a dielectric grating, the fabrication of which can be achieved by standard processing tools, and which do not lead to parasitic losses. The reason is that the thin film supports guided modes in which the light propagates parallel to the layer, increasing the path length and hence the absorption. However, guided modes are not directly accessible from the surrounding medium because of phase matching constraints-gratings can act as couplers into guided modes, allowing direct external excitation. We consider TEpolarized light at normal incidence and a film layer with a volume grating with period $d=660 \mathrm{~nm}$ and a fill 
fraction of $50 \%$. Figures $2 \mathrm{~b}-2 \mathrm{~d}$ show the effect of an increasingly stronger grating. For the high refractive index layer $n_{h}=n^{\prime}+i n^{\prime \prime}$, while for the lower $n_{l}=1+\alpha\left(\left(n^{\prime}-1\right)+i n^{\prime \prime}\right)$, providing a linear interpolation between air $(\alpha=0)$ and $n_{h}(\alpha=1)$, in which case the film is uniform. Figures 2b-d show results for $\alpha=0.3,0.1$ and 0 , respectively. Note that Fig. $2 \mathrm{~b}$ shows the solution also present in uniform structures, but shifted because the average refractive index of the layer has decreased, but also two new solutions near the real axis, due to the excitation of TE waveguide modes. The two solutions are associated with the gratings' first and second reciprocal lattice vectors. As the grating gets stronger (Figs 2c and 2d), the solution associated with a uniform film moves out of the frame, and the two grating-induced solutions become stronger and reach perfect absorption. Note that the required refractive index of the absorber lies close to the real axis, corresponding to a weakly absorbing material. Even though in this example $n^{\prime} \approx 4$ for the first grating resonance is required, by adjusting the period and other parameters this value can be reduced.
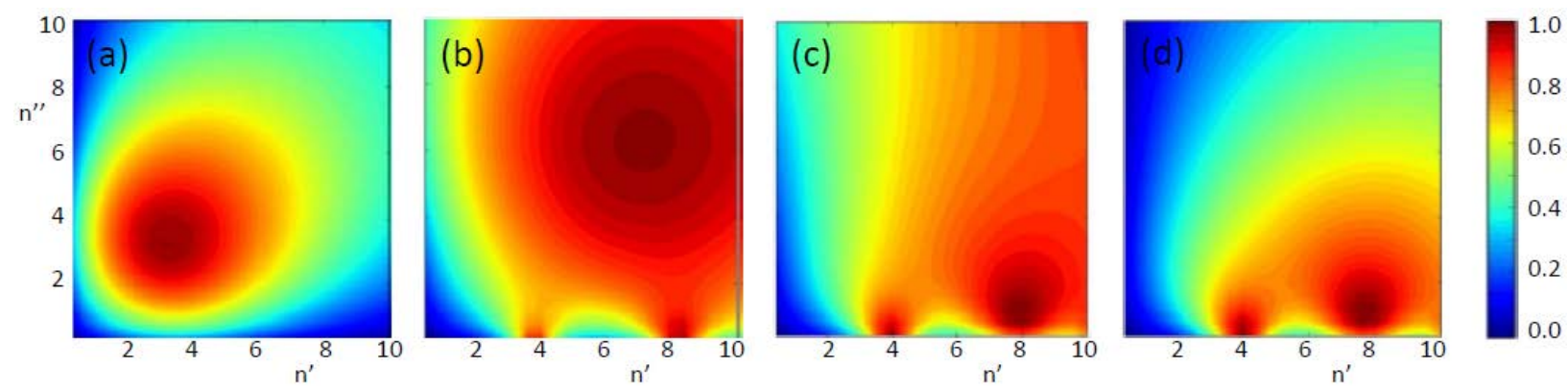

Figure 1: Absorption versus $\boldsymbol{n}^{\prime}$ and $\boldsymbol{n}^{\prime \prime}$ in four different cases for normally incident TE-polarized light of wavelength $\lambda=$ $\mathbf{7 0 0} \mathrm{nm}$ light in a layer with $\boldsymbol{d}=\mathbf{1 0} \mathrm{nm}$ in air versus $\boldsymbol{n}^{\prime}$ and $\boldsymbol{n}^{\prime \prime}$. (a) Uniform layer. (b)-(d) are for increasingly stronger volume gratings with period $\boldsymbol{\Lambda}=\mathbf{6 6 0} \mathrm{nm}$ and a fill fraction of 50\%. The high-index medium has refractive index $\boldsymbol{n}=\boldsymbol{n}^{\prime}+\boldsymbol{i} \boldsymbol{n}^{\prime \prime}$ whereas the low index medium has refractive index $\boldsymbol{n}_{l}=\mathbf{1}+\boldsymbol{\alpha}\left(\left(\boldsymbol{n}^{\prime}-\mathbf{1}\right)+\boldsymbol{i} \boldsymbol{n}^{\prime \prime}\right)$. (b) $\boldsymbol{\alpha}=\mathbf{0 . 3}$; (c) $\boldsymbol{\alpha}=\mathbf{0 . 1}$; (d) $\boldsymbol{\alpha}=\mathbf{0}$.

\section{Discussion and conclusions}

The structures discussed here lead to perfect absorption with materials with realistic refractive indices which do not suffer from parasitic absorption, and which can be fabricated using standard processing tools. Even though the analysis in this work was purely numerical, we have an analytic model which gives results consistent with those presented here. While we only considered TE polarization calculations for TM-polarized incident light show that novel solutions occur near the imaginary axis, corresponding to metallic media. We believe that polarization insensitive performance can be achieved in crossed gratings.

All calculations in this work were carried out using the freely available EMUstack package [8-9].

\section{Acknowledgements}

This work was funded by the Australian Renewable Energy Agency and the Australian Research Council through Discovery Grant DP130101009 and through the CUDOS Centre of Excellence Grant CE110001018.

\section{References}

[1] G. Bouchitté and R. Petit, “On the concepts of a perfectly conducting material and of a perfectly conducting and infinitely thin screen,” Radio Science 24, 13-26 (1989). .

[2] A. Yariv, "Critical coupling and its control in optical waveguide-ring resonator systems”, IEEE Phot. Techn. Lett., vol. 14 483-485, 2002.

[3] H. A. Haus, "Waves and Fields in Optoelectronics” (Prentice Hall, Englewood Cliffs, 1983) Ch. 7.

[4] C. Hägglund, S. P. Apell, and B. Kasemo, "Maximized optical absorption in ultrathin films and its application to plasmon-based twodimensional photovoltaics,” Nano Lett, 10, 3135-3141 (2010).

[5] M. A. Kats, et al, “Ultra-thin perfect absorber employing a tunable phase change material,” Appl. Phys. Lett. 101, 221101 (2012).

[6] S. Thongrattanasiri, F. H. L. Koppens, and F. Javier García de Abajo, “Complete optical absorption in periodically patterned graphene,” Phys. Rev. Lett. 108, 047401 (2012).

[7] C. M. Watts, "Metamaterial electromagnetic wave absorbers” Adv. Mater. 24, OP98-OP120 (2012).

[8] K.B. Dossou et al, "Modal formulation for diffraction by absorbing photonic crystal slabs," J. Opt. Soc. Am. A, 29, 817-831 (2012).

[9] See http://emustack.com 